\title{
Performance analysis of GPS / Radar integrated navigation system
}

\author{
Gwang Hee Jo ${ }^{1}$, Jin Hyuk Lee ${ }^{1}$, Jae Hee $\mathrm{Noh}^{1}$, Ju Hyun $\mathrm{Lee}^{2}$, Jae Min Ahn ${ }^{3}$, and Sang Jeong Lee,", \\ ${ }^{1}$ Department of Electronics Engineering, Chungnam National University, 34134 Daejeon, Korea \\ ${ }^{2}$ Radio Navigation, Navcours, 34014 Daejeon, Korea \\ ${ }^{3}$ Department of Radio and Information Communications Engineering, Chungnam National University, 34134 Daejeon, Korea
}

\begin{abstract}
GPS is used in various navigation fields. However, visibility is lowered in such an environment as urban canyon, and navigation performance deteriorates due to the visibility, multipath effect. So, in order to improve navigation performance in this environment, methods of integration with other sensors have been studied. In this paper, we use radar to measure the range of the landmark with known position. An integration algorithm is designed using GPS pseudorange and radar measurement.
\end{abstract}

\section{Introduction}

The Global Positioning System (GPS) transmits Pseudo Random Noise (PRN) codes using Code Division Multiple Access (CDMA). The PRN code is a ranging code used to measure the pseudorange between the satellite and the receiver. The pseudorange is calculated by the position vector of the satellite and the receiver, and the clock offset. Therefore, to solve for four variables, the receiver must receive at least four satellite signals [1]. When a receiver is located in urban canyon, satellite signals may be blocked by buildings or trees. When the number of visible satellites is insufficient, the position of the receiver can not be calculated as a general method. So we fix the altitude or receiver clock offset to reduce the number of variables we need to determine and get navigation solution. Alternatively, the position of the receiver is determined using sensors capable of providing navigation information such as an Inertial Navigation System (INS), dead-reckoning (DR), and altimeter [2-3]. In this paper, we use a radar to measure the range to a known landmark. Then, an integration algorithm is designed using GPS pseudorange and radar measurement. The simulation setup is as follows. We collect satellite signals using NI Universal Software Radio Peripheral (NI USRP). The radar signal is generated by randomly assuming the relative position of the detected landmark. Navigation performance will be shown as CEP and compared with navigation results using ublox.

\section{Calculating Radar Target Position}

The method of obtaining the azimuth angle and the elevation angle of the target is shown in Fig. 1. Elevation angle is the angle between xy-plane and radar.

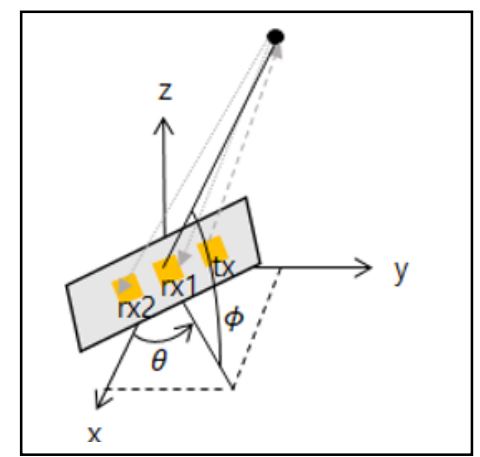

Fig. 1. Position recognition of three dimensional space target using radar

The method of calculating the azimuth angle of the target using two antennas is shown in Fig. 2.

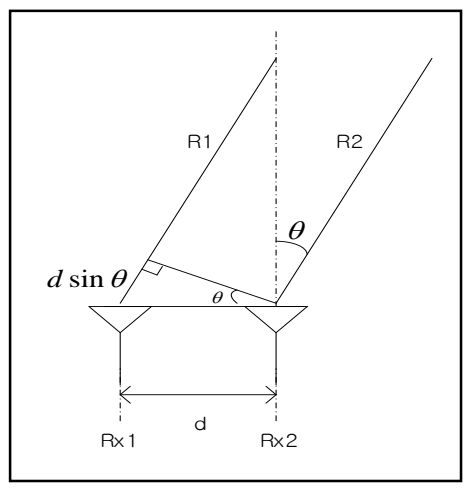

Fig. 2. Generating azimuth angle of a target

The reflected signal from the target arrives by moving R1 and $\mathrm{R} 2$ to the two antennas, which are separated by $\mathrm{d} \mathrm{cm}$. The distance between the antennas is in $\mathrm{cm}$, so if the range 
of the target is a few meters or tens of meters, it can be assumed that the signals arriving at the two antennas come in parallel.

The angle between the normal to the antenna and the target direction is $\theta$. Then the distance difference between the two paths is $d \sin \theta$. The phase of the signal coming in through the two paths can be calculated using $\phi=\operatorname{ATAN}(Q, I)$. The phases of the received signals are $\phi_{1}, \phi_{2}$, respectively. Then the phase difference of the two signals is $\phi_{\text {diff }}=\phi_{1}-\phi_{2}$. The incident angle of the signal can be calculated as a phase difference and is expressed by the following equation [4]:

$$
\theta=\operatorname{ASIN}\left(\frac{\lambda \phi_{\text {diff }}}{2 \pi d}\right) \text {. }
$$

We know the Azimuth angle, the elevation angle and the range, so we can calculate the relative position of the target.

\section{GPS/Radar Integration}

The pseudorange measurement for calculating user position $\left(x_{u}, y_{u}, z_{u}\right)$ and clock offset $\left(t_{u}\right)$ is

$$
\rho_{j}=\left\|\mathbf{s}_{j}-\mathbf{u}\right\|+c t_{u},
$$

$\mathrm{j}$ is the satellite's index [1].

If we expand equation (2) by using satellite position, user position and clock offset, it is

$$
\rho_{j}=\sqrt{\left(x_{j}-x_{u}\right)^{2}+\left(y_{j}-y_{u}\right)^{2}+\left(z_{j}-z_{u}\right)^{2}}+c t_{u} .
$$

$x_{j}, y_{j}, z_{j}$ is the jth satellite's position in three dimensions. Linearization of this equation results in

$$
\begin{aligned}
\rho_{j}= & \hat{\rho}_{j}-\frac{x_{j}-\hat{x}_{u}}{\hat{r}_{j}} \Delta x_{u}-\frac{y_{j}-\hat{y}_{u}}{\hat{r}_{j}} \Delta y_{u} \\
& -\frac{z_{j}-\hat{z}_{u}}{\hat{r}_{j}} \Delta z_{u}+c t_{u}
\end{aligned} .
$$

To simplify equation (4), we use the following equation:

$$
\begin{gathered}
\Delta \rho=\hat{\rho}_{j}-\rho_{j} \\
a_{x j}=\frac{x_{j}-\hat{x}_{u}}{\hat{r}_{j}}, a_{y j}=\frac{y_{j}-\hat{y}_{u}}{\hat{r}_{j}}, a_{z j}=\frac{z_{j}-\hat{z}_{u}}{\hat{r}_{j}} .
\end{gathered}
$$

Then equation (4) becomes

$$
\Delta \rho_{j}=a_{x j} \Delta x_{u}+a_{y j} \Delta y_{u}+a_{z j} \Delta z_{u}-c t_{u} .
$$

The four satellite measurements are represented by

$$
\Delta \boldsymbol{\rho}_{G P S}=H_{G P S} \Delta \mathbf{x}_{G P S} \cdot
$$

$$
\begin{gathered}
\Delta \boldsymbol{\rho}_{G P S}=\left[\begin{array}{c}
\Delta \rho_{1} \\
\Delta \rho_{2} \\
\Delta \rho_{3} \\
\Delta \rho_{4}
\end{array}\right], \Delta \mathbf{x}=\left[\begin{array}{c}
\Delta x_{u} \\
\Delta y_{u} \\
\Delta z_{u} \\
-c \Delta t_{u}
\end{array}\right], \\
H_{G P S}=\left[\begin{array}{llll}
a_{x 1} & a_{y 1} & a_{z 1} & 1 \\
a_{x 2} & a_{y 2} & a_{z 2} & 1 \\
a_{x 3} & a_{y 3} & a_{z 3} & 1 \\
a_{x 4} & a_{y 4} & a_{z 4} & 1
\end{array}\right] .
\end{gathered}
$$

Radar range can be expressed as Eq. (8). The difference between radar and GPS is that the clock offset does not affect the radar range measurements. Therefore, the $\mathrm{H}$ matrix of Radar is given by

$$
H_{\text {Radar }}=\left[\begin{array}{cccc}
a_{x 1} & a_{y 1} & a_{z 1} & 0 \\
a_{x 2} & a_{y 2} & a_{z 2} & 0 \\
a_{x 3} & a_{y 3} & a_{z 3} & 0 \\
a_{x 4} & a_{y 4} & a_{z 4} & 0
\end{array}\right],
$$

$a_{x j}, a_{y j}, a_{z j}$ is defined in the same way as Eq. (6). Radar measurements are expressed as

$$
\Delta \boldsymbol{\rho}_{\text {Radar }}=H_{\text {Radar }} \Delta \mathbf{x} .
$$

We combine equation (8) and (11) to form

$$
\left[\begin{array}{c}
\Delta \boldsymbol{\rho}_{G P S} \\
\Delta \boldsymbol{\rho}_{\text {Radar }}
\end{array}\right]=\left[\begin{array}{c}
H_{G P S} \\
H_{\text {Radar }}
\end{array}\right]\left[\begin{array}{c}
\Delta x_{u} \\
\Delta y_{u} \\
\Delta z_{u} \\
-c \Delta t_{u}
\end{array}\right] .
$$

We can use the least square method to navigate using equation (12).

\section{Simulation}

GPS signals were collected using NI USRP, and Radar transmit and receive signals were generated by Software. The radar was assumed to have a center frequency of 24 $\mathrm{GHz}$ and a bandwidth of $250 \mathrm{MHz}$. The landmark was set to one. Range and direction were defined as $58.2 \mathrm{~m}$, azimuth 314, elevation 26. Initially, navigation is performed using only four satellites. After 10 seconds, only 3 satellite signals were used and combined with Radar measurements. Navigation results are shown in Fig. 3. Navigation results using the measurements provided by the ublox receiver are shown in Fig. 4. This is the result of postprocessing using rtklibrary. Both navigation results use the Earth-Centered Earh-Fixed (ECEF) coordinate system.

The vector and matrix are 


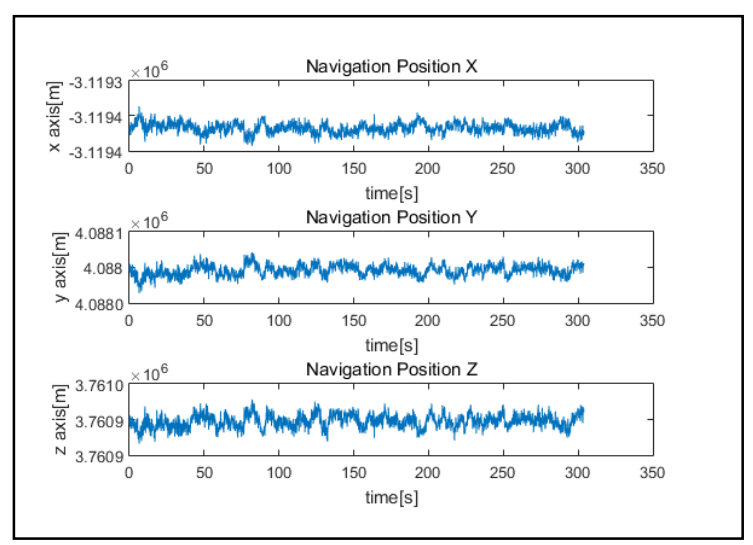

Fig. 3. Navigation results using GPS and Radar (ECEF)

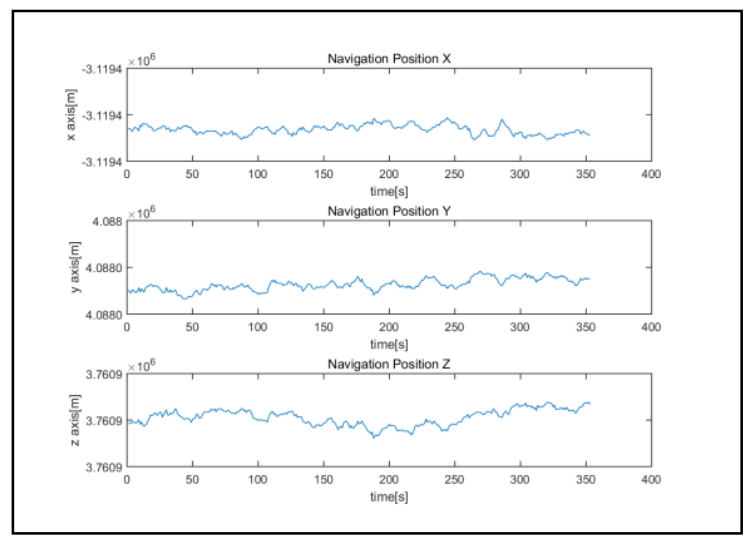

Fig. 4. Navigation results using ublox (ECEF)

The results of the two translations into the ENU coordinate system are shown in Fig. 5 and Fig. 6. When the visible satellite is not enough, the radar detects a landmark whose position is known and calculates a range. As a result, the receiver can navigate as if it received one more satellite signal. When radar is used as an assistant sensor, navigation performance deteriorates compared to postprocessing results using ublox receivers. However, since the CEP is $3.311 \mathrm{~m}$, radar can be used as a navigation assistant sensor when the visible satellite is not enough.

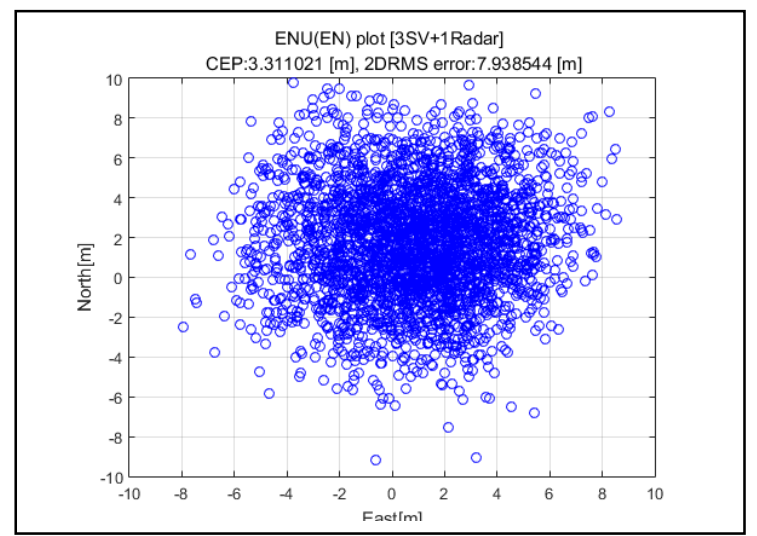

Fig. 5. Navigation results using GPS and Radar (ENU)

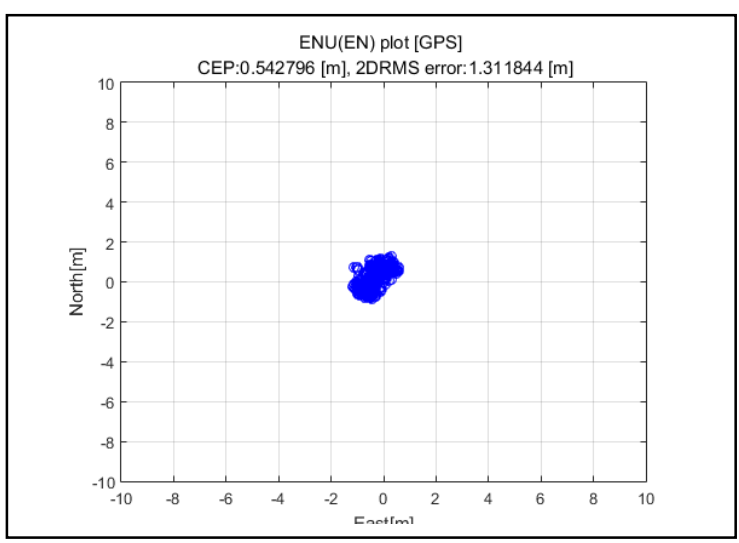

Fig. 6. Navigation results using ublox (ENU)

\section{Conclusions}

In this paper, we have designed an algorithm that integrates GPS and radar. When there were three visible satellites, we navigated using radar measurements. The results showed a CEP available for navigation. As further work, we will use the Kalman filter instead of the least square method, and consider how to compensate for the error in feedback.

\section{Acknowledgments}

This work has been supported partly by the National GNSS Research Center Program of Defense Acquisition Program Administration and the Agency for Defense Development and also supported by the Research Fund of Chungnam National University

\section{References}

1. E.D.Kaplan, C.J. Hegarty, Understanding GPS (2006)

2. D.B.Cox, Navigation, Jounal of the institute of Navigation, 25, 2 (1978)

3. T.N.Upadhyay, S.Cotterill, A.W. Deaton, IEEE TRANSACTIONS ON Aerospace\&Electronic Systems, 29, 3 (1993)

4. H.K. Sim, M.A. Jung, The Journal of Korean Institute of Communications and Information Sciences, 40, 07 (2015) 